\title{
Das Versagen von Politik und Medien im Golf-Krieg. Ein Rückblick auf ein bedrückendes Kapitel der 'Medienwirklichkeit'
}

\author{
von Rüdiger Schlaga
}

1856 erließ der britische General Sir William Codrington einen Befehl, der vorschrieb, wie Kriegsberichterstatter von nun an zu berichten hatten und vor allem, worüber nicht - die moderne Militärzensur erblickte das Licht der Welt. Seitdem gehört sie zu den Standards eines jeden Krieges, so auch im zweiten Golf-Krieg. ${ }^{1}$ Wieder war die Pressefreiheit einer der Verlierer des Krieges, noch bevor die ersten Luftangriffe von den Alliierten am 17. Januar 1991 geflogen wurden. Im Irak hatte es Pressefreiheit auch vorher nicht gegeben. Alle an der Anti-Irak-Allianz beteiligten Länder verpflichteten noch im Januar jedoch gleichfalls ihre Journalisten vor Ort und, wo möglich, in den Redaktionen zuhause auf umfangreiche Grundregeln zur Berichterstattung. Kritik an diesem Vorgehen wiesen die Behörden zurück. Denn, so beispielsweise ein Sprecher des britischen Verteidigungsministeriums, „eine Zensur gibt es bei uns nicht". Es handele sich vielmehr lediglich um „Orientierungshilfen“ für Redakteure und „Verhaltensmaßregeln" für Reporter.

\section{Die Zensurbestimmungen im Golfkrieg}

Wir erinnern uns: Kein Reporter durfte unbeaufsichtigt vor Ort recherchieren und von dort berichten. Von den Verteidigungsministerien ausgewählte 7 bis 18 Berichterstatter wurden vielmehr in sogenannten Pools zusammengefaßt, in Uniformen gesteckt und unter Anleitung eines Presseoffiziers an die von Militärs ausgesuchten Orte und Frontabschnitte gebracht. Zusätzlich stellten die Militärpressestellen die Pools für jeden dieser Ausflüge neu zusammen. Den Reportern war es damit unmöglich, Veränderungen festzustellen und verschiedene Informationen vor Ort miteinander zu vergleichen; das aber wäre angesichts der völlig undurchsichtigen Informationslage für eine objektivere Berichterstattung unabdingbar gewesen. Von den etwa 1.500 nach Saudi Arabien entsandten Reportern waren insgesamt lediglich ca. $120 \mathrm{zu}$ den Pools zugelassen. Die Zurückgewiesenen blieben auf die Berichte der Pool-Mitglieder, die Pressekonferenzen des General Schwarzkopf und des Pentagon in Washington sowie die Informationen angewiesen, die weltweit von $\mathrm{CNN}$ verbreitet wurden. Journalisten, die aus Ländern kamen, die nicht der militärischen Anti-Irak-Allianz angehörten, wurden nicht zu den Pools zugelassen. Kein einziger deutscher Korrespondent war jemals persönlich im Aufmarschgebiet anwesend. Die Auswahl der US-Journalisten erfolgte zudem auch danach, welches Ansehen sie bei den Militärs genossen. Nach Angaben der liberalen US-amerikanischen Pressebeobachtungsorganisation „Fairness and Accuracy in Reporting" (FAIR) erhielt kein einziger freier oder ausdrücklich kriegskritischer Journalist Zugang zu den

Dr. phil. Rüdiger Schlaga war bis Ende 1991 Presse- und Öffentlichkeitsreferent der Hess. Stiftung Friedens- und Konfliktforschung (HSFK) in Frankfurt/Main 
Pools. Teilweise wurde diesen sogar die Akkreditierung für eine Berichterstattung aus Saudi Arabien völlig verwehrt.

Die militärische Führung handhabte die Pool-Praxis sowie die anderen Zensurregularien äußerst effizient. Ein hoher Armeeoffizier beschrieb die Ausgangsbasis der Anstrengungen des Pentagon, die Berichterstattung zu kontrollieren: „Es gibt einige Leute, die sagen, die Medien seien der Feind. Doch sie sind tatsächlich eher ein Schlachtfeld. Darauf müssen wir gewinnen." ${ }^{22}$ Und das Militär gewann auch den Krieg gegen die Medien und die Pressefreiheit. Norman Schwarzkopf, US-General und Oberster Kommandierender der Alliierten Streitkräfte am Golf, erklärte nach dem Ende der Kampfhandlungen stolz, wie erfolgreich die Pool-Journalisten manipuliert worden seien. Entgegen ihrer Annahme seien sie nämlich weder im Westen an der Front gewesen, noch hätten sie jemals die saudisch-irakische Grenze zu Gesicht bekommen. Ihnen sei vielmehr jedesmal derselbe Frontabschnitt vorgeführt worden. ${ }^{3}$

Zudem wurden alle Berichte und Foto- wie Filmaufnahmen der Poolmitglieder, bevor sie den anderen Journalisten oder den Heimatredaktionen übermittelt werden durften, einer sogenannten Sicherheitsüberprüfung unterzogen. Die Weitergabe wurde oft verzögert. Die Zensuroffiziere konnten die Übermittlung von Informationen und Bildmaterial aus "Sicherheitsgründen" untersagen, wenn sie das „nationale Interesse“ gefährdet sahen. Sowohl „Sicherheit" als auch das "nationale Interesse" sind dabei äußerst dehnbare Vokabeln - jeder Eingriff läßt sich damit begründen.

Für jeden Journalisten nachvollziehbar ist zweifelsohne, daß es sinnvoll sein kann, über Einzelheiten, die für den Gegner von militärischen Wert sein könnten, nicht zu berichten. Entsprechend plausible Regeln sind weitgehend akzeptiert. Kritik us-amerikanischer Medienvertreter galt dagegen vor und während des Golf-Krieges neben der Poolregelung und der daraus folgenden mangelnden Bewegungsfreiheit dem Passus 12 der US-"Groundrules". Darin heißt es u.a., daß über „Details größerer Kampfschäden oder größerer Menschenverluste einer spezifischen US- oder Koalitionseinheit" nicht berichtet werden dürfe, „solange diese Information einen taktischen Vorteil für den Feind bedeuten kann und noch nicht vom US-Hauptquartier freigegeben worden ist". Der Umfang der Zerstörung und das Ausmaß an menschlichen Opfern darf nicht präzise benannt werden. Die Bestimmungen sehen statt dessen die Kategorien „leicht“, „mäßig“" und „schwer" vor. ${ }^{4}$ Mit anderen Worten: Ohne ausdrückliche Genehmigung durch die Militärs sind für die Bevölkerung daheim genaue Informationen über Tote, Verletzte und materielle Schäden tabu. Verboten war es aber auch, über die Auswirkungen der alliierten Angriffe auf den Irak und dessen Truppen in Kuwait sowie Einzelheiten über das Vorgehen der gegnerischen Truppen oder über deren Opfer zu berichten. ${ }^{5}$ Es galt, bei der Bevölkerung daheim den Eindruck zu wahren, es handele sich um einen "sauberen" Krieg, der ohne Zerstörung, ohne Blutvergießen und ohne den Verlust von Menschenleben erfolgreich abläuft. Bilder von toten Menschen und völlig zerschlagenen Städten hätten die Illusion gestört, ihr die Grundlage geraubt. Zu dieser Sterilisierung von Krieg gehörte als eine der ersten Maßnahmen des US-Verteidigungsministeriums, die militärischen Zeremonien bei der Anlandung der in Leichen- 
säcken eingeflogenen gefallenen US-Soldaten auf der Luftwaffenbasis Dover im Staate Delaware abzusagen. Es sollte vermieden werden, die sich womöglich häufenden Fälle immer wieder über die Fernsehschirme flimmern sehen zu müssen.

Die US-Militärs waren keineswegs zimperlich, wenn es darum ging die Zensurregularien durchzusetzen. Reporter, die versuchten eigenständig zu recherchieren, wurden massiv unter Druck gesetzt und mit Ausweisung bedroht. Der New Yorker „Fund for Free Expression“ listet in einem Bericht über die Einschränkungen im Golf-Krieg Vorfälle auf, bei denen Journalisten mit der Waffe bedroht, in Arrest genommen und ihnen die Akkreditierung entzogen wurde. ${ }^{6}$

\section{Zensur - Beruhigungsmittel für die eigene Bevölkerung}

Die US-Militärs und ihre politische Führung machen kein Geheimnis daraus, daß die Angst vor militärischem Geheimnisverrat nicht das entscheidende Motiv für die Zensur war. Ihnen war es vielmehr darum gegangen, den „Krieg in der Wohnstube", wie zu Zeiten des Vietnamkrieges, sich nicht wiederholen zu lassen. Nicht noch einmal sollte - so die US-amerikanische Dolchstoßlegende zum Vietnamkrieg - ein Krieg wegen der mangelnden Unterstützung an der „Heimatfront“, vor den heimischen Fernsehgeräten verloren werden.

Deshalb bedurfte es einer konzertierten Aktion von Militär und Politik, um die öffentliche Meinung in den USA unter Kontrolle zu halten. Es galt mit allen Mitteln, einen als jederzeit möglich befürchteten Stimmungsumschwung im Lande zu verhindern. Eines der wichtigsten Instrumente der Regierung dazu war die Zensur. Mit ihrer Hilfe kontrollierten sie alle Informationen für die Öffentlichkeit. Es war somit keine militärisch notwendige Zensur, sondern im wesentlichen eine politische, die, so der Kommentator der International Herald Tribune, William Pfaff, allein „die Aufgabe hatte, den Krieg politisch akzeptabel zu halten, insbesondere gegenüber der amerikanischen Öffentlichkeit." ${ }^{\text {"7 }}$

Die ehemals jungen Offiziere aus dem Vietnamkrieg und heutigen hochdekorierten Generäle haben ihre Lektion gelernt. „Es wird diesmal keine Diskussion über die Kriegskosten geben", stellte einer von ihnen noch vor Beginn des Luftkriegs klar. „Deshalb kann die Tendenz einer jeden Demokratie zu debattieren, nicht gegen uns arbeiten. ${ }^{\text {" }}{ }^{8}$ Und so wurde die Öffentlichkeit vorsätzlich belogen und organisiert in die Irre geführt. Scharf kritisiert die Süddeutsche Zeitung die Kumpanei von Militärs und Politikern in der Zensurfrage: „Wenn man es für nötig hält, sind Vertuschung und Lüge die Fortsetzung der Politik mit anderen Mitteln“.9?

Die Eingriffe der Militärzensoren beschränkten sich nicht allein auf das Schlachtfeld. Im Dezember 1990 veröffentlichte der Hanau Herald, die Regionalzeitung der US-Garnison östlich von Frankfurt, Anweisungen, wie sich die Soldaten im Umgang mit der Presse verhalten sollten. Darin heißt es u.a.: ,Wenn Sie ein Interview geben möchten, wird ein Verbindungsoffizier das Gespräch gerne 
koordinieren, damit alles glatt verläuft. ... Gehen Sie sicher, daß Ihre Antworten keine Sicherheitsbestimmungen verletzen. Sagen Sie die Wahrheit, aber achten Sie darauf, was Sie sagen. Denken Sie dabei auch an das Wohl des Militärs. "

Die politische und militärische Führung der USA fürchtete in der Tat niemals, sie könnte den Krieg militärisch verlieren. Der "Sieg" war nur eine Frage des eingesetzten Potentials und der Zeit. Doch gerade die Zeit, d. h. ein sich möglicherweise hinziehender Krieg hätte neue Fragen aufwerfen können. Dies machte die Lage innenpolitisch latent labil. Niemals konnte die Administration vor einer politischen Niederlage sicher sein. Bei Anhörungen im Senat im November und Dezember 1990 sprachen sich beispielsweise zehn von zwölf früheren US-Verteidigungsministern dafür aus, die Sanktionen gegen den Irak wirken zu lassen und nicht vorschnell Krieg zu führen. Auch die Abstimmung im Senat noch wenige Tage vor Ablauf des UNO-Ultimatums und trotz des weit fortgeschrittenen Truppenaufmarsches in der saudischen Wüste war denkbar knapp: $53: 47$.

\section{Demokratie und Zensur gehen nicht zusammen}

Es ist inakzeptabel, wenngleich kaum anders zu erwarten, daß ein diktatorisches System wie das des Irak Zensur praktiziert. In einer Demokratie jedoch ist Zensur nicht hinnehmbar, selbst wenn sie von der Mehrheit des Souveräns, der eigenen Bevölkerung, gewünscht wird. ${ }^{11}$

Sicher stellt es für eine Demokratie ein Problem dar, daß ihre Regierung niemals ungestraft mit dem Leben ihrer Bürger spielen kann. Doch die Antwort auf die Erkenntnis, ein US-Präsident bekäme größte innenpolitische Probleme, wenn 10.000 US-Soldaten im Krieg fielen, während der Diktator Hussein unberührt zwei bis drei Millionen Iraker opfern könne, kann nicht Zensur heißen. Denn der (scheinbare) Nachteil des Westens ist ein Preis des historische Fortschritts, den die demokratische Gesellschaft gegenüber einer diktatorischen zu zahlen hat. Diesen Nachteil dann doch mit Hilfe der Zensur aufheben zu wollen, bedeutet nichts anderes als die Demokratie wieder in Frage zu stellen. Zu Recht betont Michael Kinsley, Kommentator im Time Magazine, wenn es Saddam Hussein mit seiner Propaganda gelänge, die Mehrheit der US-Bürger zu überzeugen, daß Krieg eine schlechte Idee sei, dann müsse dieser Krieg eben beendet werden. „Wenn Sie (die Leser - R.S.) nun befürchten, daß Diktaturen deshalb in den internationalen Beziehungen einen unfairen Vorteil haben, dann zürnen sie mit der Demokratie und nicht mit dem Journalismus. ${ }^{\text {12 }}$

Zensur steht im fundamentalen Gegensatz zu den Prinzipien einer demokratischen Gesellschaft: Transparenz von staatlichen Entscheidungen, Informationen über deren Folgen und Partizipation der Bürger an ihrer Umsetzung. Deshalb war auch eine der Hauptforderungen der Bürgerrechtsbewegungen in den früheren sozialistischen Staaten die nach Presse- und Informationsfreiheit, gerade in Abgrenzung zu den totalitären Systemen. Alle im Westen haben damals applaudiert. Doch im Golf-Krieg wurden diese Freiheiten von Militärs und Politikern leichtfertig außer Kraft gesetzt. Damit war der Golf-Krieg gleichzeitig 
ein Krieg gegen das Recht der Menschen auf freie Information. Denn im Ersten Zusatzartikel der US-Verfassung heißt es unumwunden: „Der Kongreß darf kein Gesetz erlassen, das ... die Rede und Pressefreiheit ... einschränkt.“ Artikel 5 des Grundgesetzes stellt lapidar fest: „Eine Zensur findet nicht statt." Alle bundesdeutschen Behörden sind vielmehr verpflichtet, auf Anfrage alle notwendigen Informationen herauszugeben, ungeachtet möglicher politischer Folgen. ${ }^{13}$

Demokratische Gewaltenteilung beruht nicht zuletzt in der Erkenntnis, daß unkontrollierte Macht die Tendenz hat, sich zu verselbständigen und sich gegen die Demokratie zu wenden. Die politische Exekutive, die Regierung wie das Militär, kann nur von einem debattierenden Parlament und einer informierten Öffentlichkeit kontrolliert werden. Beide brauchen dafür funktionierende Medien. Blindes Vertrauen in die fortwährende Rechtschaffenheit der Exekutive gehört nicht zum Bestand einer demokratischen Verfassung oder Gesellschaft.

\section{Die deutschen Medien: Opfer und Täter zugleich}

Wenn es bei dem Eindruck bliebe, die Medien seien nur Opfer der Manipulation durch Politik und Militär, so wäre dies sicherlich falsch. Während der Golf-Krise und des Krieges spielten sie eine Doppelrolle. Sie waren Opfer und Täter zugleich.

So sehr es gilt, die Militärzensur als Verstoß gegen demokratische Prinzipien anzuprangern, so sehr ist aber auch allgemein bekannt, daß sie, mit wenigen Ausnahmen, noch immer Teil moderner Kriege war und ist. Sie konnte deshalb nicht überraschend gewesen sein, schon gar nicht vor dem Hintergrund der Erfahrungen, die die US-Medien während der letzten US-Interventionen in Grenada und Panama mit der Pentagon-Zensur oder die britischen mit der ihres Militärs 1982 während des Krieges gegen Argentinien um die Falkland/Malvinas-Inseln gewonnen hatten.

Spätestens seitdem der UNO-Sicherheitsrat Irak das Ultimatum zum 15. Januar gesetzt hatte, Kuwait zu räumen, hätte man sich gezielt auf den Tag X, den Krieg und die Zensur, vorbereiten können und müssen. Wo aber waren die Strategien und die handwerklichen sowie inhaltlichen Vorbereitungen, sich gegen die Zensur zur Wehr zu setzen, sie zu unterlaufen? Nikolaus Brender, Leiter der Auslandsredaktion des WDR, gibt jedoch im nachhinein offen zu: „Die ARD war nicht vorbereitet. "14

Während in vielen der Print-Medien erfolgreich versucht wurde, den Anteil an selbstrecherchierter, analysierender Hintergrundberichterstattung über das Umfeld des Krieges auszubauen, war dies vor allem im Fernsehen in der Regel nicht der Fall. Es fehlte an tiefergehenden Analysen.

Klaus Bresser, Chefredakteur des ZDF gestand in ehrenwerter, gleichwohl beschämender Weise ein, daß das ansonsten so omnipotent agierende Medium Fernsehen in der Krise keine vertiefende, hinterfragende Analyse und Berichterstattung leisten könne: „Wer sich noch ausführlicher informieren will, 
für den gibt es die Tageszeitungen, Wirtschaftsblätter, politischen Magazine. Das Fernsehen ist ein Medium unter vielen. Es kann nicht alles allein leisten. Es ist nach dem Hörfunk das schnellste Medium, es bedarf der Erläuterung durch das gedruckte Wort." ${ }^{15}$ Dem US-Fernsehsender CNN, der sich generalstabsmäßig auf die Berichterstattung vom Kriegsschauplatz vorbereitet hatte, wurde andererseits vorgeworfen, lediglich zynisch den Krieg als Ware zu vermarkten. Gleichwohl wurde das Cable News Network - nicht zuletzt von den Korrespondenten der deutschen elektronischen Medien - regelmäßig und oft unausgewiesen als Quelle für ihre eigenen Berichte genutzt.

In den Tagen vor Ablauf des UNO-Ultimatums am 15. Januar dominierte ein „count-down-Journalismus“. In den zunehmend informationsärmeren Tagen nach Beginn der Kampfhandlungen produzierten speziell die elektronischen Medien mit einer hektischen Rund-um-die-Uhr-Berichterstattung den Anschein von Authentizität und objektiven Dabei-seins. Sondersendung reihte sich an Sondersendung. Allein die ARD produzierte 32 „Brennpunkte“. Das in den Vereinigten Staaten verbreitete „Infotainment" gewann im deutschen Fernsehen an Boden. Keine dieser Sendungen, insbesondere des Frühstücksfernsehens von ARD/ZDF wie auch der Privatsender, kam aus ohne ihre Militärspezialisten, Regionalexperten oder Friedensforscher. Die „Expertokratie" feierte fröhliche Urständ. Akademische Titel genauso wie militärische Rangabzeichen suggerierten Seriosität und tiefschürfende Analyse für etwas, was dann oft kaum besser war als Kaffeesatzlesen. Das aus Angst gespeiste immense Informationsbedürfnis der deutschen Bevölkerung wurde zur Rechtfertigung für die Programmacher, immer größere Sendeflächen mit kaum fundierter Information unter großem Aufwand und Showeffekt zu füllen. Die reflektierende und ruhig analysierende Reportage fiel der Hektik und der selbstformulierten Gesetzlichkeit des „Wir müssen doch informieren!“ zum Opfer. Die immens gestiegenen Einschaltquoten dienten der Rechtfertigung, sich weitgehend ungeschützt der Zensur zu unterwerfen und immer weiter zu berichten, auch wenn es nichts zu berichten gab.

Zudem zeigen die erstmals massiv zum Einsatz gekommenen neuen schnellen Informationstechniken für den reflektierenden und distanzierten Journalismus eine besondere Gefahr. Die Schnelligkeit wird zum ökonomisch begründeten Selbstzweck. Der Kampf um Marktanteile erzwingt inzwischen, jede Neuigkeit oft genug ungeprüft per Satellit in den globalen Informationsmarkt einzuspeisen.

Finanziell hat sich die erhöhte Aufmerksamkeit der Konsumenten für die Medien mit Ausnahme vielleicht von $\mathrm{CNN}$, gleichwohl nicht ausgezahlt. In Großbritannien beispielsweise stornierte British Airways alle Fernseh-Spots bis zum Ende des Krieges. Coca Cola erklärte es für „unangemessen, im Rahmen der Kriegsberichterstattung zu werben". ${ }^{16}$

Die Zensurpraxis im Golf-Krieg spitzte allerdings nur die inzwischen kaum mehr übersehbare Tendenz der alläglichen Zensur in unserer Gesellschaft zu, die zunehmend den demokratischen Anspruch auf Presse- und Informationsfreiheit ad absurdum führt. Die Zeitschrift der IG Medien „Publizistik \& 
Kunst" sowie der "Journalist" des Deutschen Journalistenverbandes veröffentlichen regelmäßig Beispiele aus der „unendlichen Geschichte Zensur“. Mal verbietet ein Bürgermeister den Abdruck eines unliebsamen Gedichts von Erich Fried in einer städtischen Broschüre; dann wieder werden der nachfragende Journalist und seine Leser beispielsweise nach einem Zwischenfall in einem Atomkraftwerk von der Werksleitung abgespeist mit dem lapidaren, informationsverweigernden Satz: „Niemals bestand eine Gefahr für die Bevölkerung." Stadtverwaltungen genauso wie Landesministerien verstehen ihre Pflicht, die Öffentlichkeit über alle Entwicklungen auf dem Laufenden zu halten, oft eher als Zwang und begnügen sich vorzugsweise damit, PR-Meldungen herauszugeben. Es scheint, als würde diese Form der alltäglichen Zensur inzwischen auch von vielen Journalisten als kaum noch abänderbar akzeptiert.

Zudem, auch in ruhigeren Zeiten als während eines Krieges ist der „investigative Journalismus", von wenigen Ausnahmen abgesehen, in Deutschland keine verbreitete Arbeitsform. Der Fehlentwicklungen in der Bundesrepublik, denen es nachzugehen gälte, gibt es viele: Die Bundeswehr und die politische Führung auf der Bonner Hardthöhe benutzen den Geheimhaltungsstempel mit Vorliebe gegen das Informationsrecht der eigenen Bürger; Fehlinformationen von Behörden in Ökologiefragen sind an der Tagesordnung; die Waffenexportpolitik der Bundesrepublik unterliegt weitgehend der Geheimhaltung, wie die gesamte Exportpolitik fortwährend verschleiert und der kritischen Überprüfung durch die Medien entzogen wird.

Der Anspruch, die Pressefreiheit auch durchzusetzen, d. h. auch die Kontrollaufgabe als „vierte Gewalt" ernstzunehmen, wird jedoch von den Medien selbst gefährdet; eigentlich wird sogar darauf verzichtet. Die reibungslose Kooperation mit den Mächtigen, die Anpassung an die Parteien- und Interessensverbändepolitik in den Rundfunkräten und das Starren auf die Einschaltquote beherrscht mehr und mehr die deutsche Medienlandschaft. ${ }^{17}$

\section{Die Zensur hat viele Gesichter}

Zensur heißt nicht nur, daß den Medien und damit dem Bürger Informationen über den Krieg vorenthalten werden. Sie hat ein vielfältigeres Gesicht. Eines davon ist das der Selbstzensur: Amerikanische Fernsehgesellschaften weigerten sich bereits in der Vorkriegszeit, Anti-Kriegsspots zu senden, so wie bundesdeutsche Zeitungen den Abdruck entsprechender Anzeigen gar nicht oder nur nach inhaltlichen Änderungen abdruckten. Die niedersächsischen Grünen beabsichtigten z. B. in der Hannoverschen Allgemeinen Zeitung und der Nordwest-Zeitung Oldenburg vier Tage vor Ablauf des UN-Ultimatums folgende Anzeige zu schalten: „Verweigert den Kriegsdienst, verlaßt die Armee! Wenn Ihr den Befehl bekommt, in einen Krieg irgendwo auf der Welt zu gehen, dann sagt nein!" Die HAZ ließ nur den Halbsatz "Verweigert den Kriegsdienst" zu. Die Nordwest-Zeitung lehnte selbst den Aufruf zur Kriegsdienstverweigerung ab, weil diese eine „schädliche Wirkung" habe. ${ }^{18}$ Die Deutsche Eisenbahnreklame weigerte sich, Werbeplakate für Kriegsdienstverweigerung auf den Bahnhöfen zu kleben, da eine solche Werbung eine aufgebrachte Stimmung 
erzeugen könne. ${ }^{19}$ Die Londoner BBC setzte John Lennons „Give Peace a Chance" und "Imagine" neben 65 weiteren Titeln auf den Index. Der Privatsender Radio Schleswig-Holstein setzte Nenas „99 Luftballons" als "naives Friedenslied" auf den Index, weil es der "ernsthaften Bedrohung am Golf" nicht gerecht werde. Doch auch vor Eingriffen in die redaktionelle Verantwortlichkeit schreckten Funkhausbürokraten nicht zurück. So setzte die BBC aus „aktuellem Anlaß“ den Film „Projekt Babylon" vom Programm ab, in dem der Beweis für weitere Verstrickungen westlicher Industrien und Regierungen beim Bau einer Superkanone des Irak geführt wurde. Auch vor Satire machten die selbsternannten Zensoren in den Funkhäusern nicht halt. Der Kabarettist Martin Buchholz verlor Anfang Februar seine regelmäßige Satirekolumne im „Morgenecho“ des SFB 2. Die Begründung von Programmdirektor Wolfgang Seifert: „Die Kriegssituation mit gesteigerten Schrecken" habe deutlich gemacht, daß Satire in der Sendezeit "mit der größten Akzeptanz" bei den Zuhörern keinen Platz habe. ${ }^{20}$

Bei den großen Fernsehgesellschaften in den USA ist eine weitere Form von (Selbst-)Zensur festzustellen. FAIR, die bereits erwähnte liberale Medienbeobachtungsorganisation, analysierte die allabendlichen Fernseh-Nachrichtengesprächsrunden von $\mathrm{ABC}$, CBS und NBC. Ihre Ergebnisse sprechen der verbreiteten Meinung, in den USA hätte es eine breite, öffentliche Debatte um das Für und Wider einer Militäraktion am Golf gegeben, Hohn: Im gesamten August 1990 waren etwa die Hälfte der Gäste frühere oder augenblickliche Regierungsbeamte. Die geladenen Experten stammten fast ohne Ausnahme von konservativen, regierungsnahen Instituten. Kein einziger Vertreter einer profilierten Friedensorganisation erhielt eine Chance aufzutreten. $98 \%$ (Nightline) bzw. $87 \%$ (MacNeil/Lehrer NewsHour) waren weiße Amerikaner, während unter den in Saudi Arabien später stationierten Truppen etwa $25 \%$ Schwarze waren. Schließlich zählte FAIR während des Krieges bei allen 878 Interviews, die von den großen Networks gesendet wurden, nur ein einziges, das von einer nationalen Friedensorganisation stammte. ${ }^{21}$ Auch die Bundeswehr übte sich ganz praktisch im Gebrauch der Zensur. Bereits anberaumte Pressekonferenzen, in denen in der betroffenen Kaserne in Schöneck bei Frankfurt über die Entsendung von Bundeswehreinheiten in die Türkei berichtet werden sollte, wurden ersatzlos gestrichen. Fragen nach der Zahl der von Verlegungen betroffener Soldaten blieben unbeantwortet. ${ }^{22}$.

\section{Der Linguizid - die Zerstörung der Sprache durch Militär und Medien}

Die verharmlosende Sprache der Militärs ist ein anderer Teil der Zensur auch in Friedenszeiten. Die Opfer kommen darin nicht vor. Die Realität wird erfolgreich verschleiert und der Krieg der Öffentlichkeit unblutig, damit akzeptabel präsentiert. Die Aufgabe der Medien, Wirklichkeit transparent zu machen, ist außer Kraft gesetzt. Nur zu regelmäßig werden die Begriffe der Militärs von Redakteuren und Korrespondenten unreflektiert übernommen und mit der Berichterstattung in die Köpfe der Rezipienten daheim transportiert. Größere Sorgfalt im Umgang mit den Agenturen, die strukturell bedingt, im besonderem Maße anfällig sind, vorgegebene Formulierungen in ihre Texte unkritisch zu übernehmen, ist als Minimum sachlicher Kriegsberichterstattung zu verlangen. Kriege sind keine Naturereignisse oder Sportveranstaltungen. Deshalb noch ein- 
mal: Kriege brechen nicht aus, sie werden gemacht. In Kriegen gibt es keine Erfolge und Sieger, es gibt lediglich Opfer und Zerstörung. Auch Siegesfeiern und Konfettiparaden vermögen daran nichts zu ändern.

Einige weitere Beispiele für den manipulativen Gebrauch von Sprache: Ganz in der Tradition des Orwell'schen Newspeak ist heute bei der US-Armee nicht mehr von Särgen oder Leichensäcke die Rede, sondern von „menschlichen Überreste-Kästen“ bzw. „-Taschen“. Im Irak wurden Atomreaktoren „lahmgelegt, auf Jahre hinaus unbrauchbar gemacht". Folgt man den offiziellen Verlautbarungen, dann ist bei den Angriffen keine „nennenswerte" Radioaktivität in die Atmosphäre gelangt. Das gleiche gilt für Chemiewaffen und biologische Kampfstoffabriken. Sie wurden lediglich „ausgeschaltet" und damit „unschädlich" gemacht. Menschen werden als "weiche" Ziele „ausgeschaltet" oder "neutralisiert", wenn sie getötet werden. Städte oder Militärstellungen sind "harte" Ziele, die "ausradiert" werden. Von „Feuerzauber" ist in den Life-Berichten aus Bagdad die Rede. Wenn es gelungen war, eine Elite-Einheit „auszuschalten“, dann wird kaum das Bild von 10.000 getöteten oder verwundeten Männern vor dem inneren Auge des Hörers oder Lesers der Nachricht auftauchen. B-52Bomber überzogen das Land vorgeblich zielgenau mit Bombenteppichen. Auch wenn man von den Kuscheligkeit suggerierenden „Teppichen" hier einmal absieht, so haben die schließlich doch zugestandenen vielen Fehlwürfe lediglich „Kollateralschäden“ zur Folge, so der gebrauchte Fachbegriff für das ebenfalls die realen Auswirkungen verharmlosende deutsche Wort Nebenschäden. ${ }^{23}$ Im übrigen trägt eine Definition der Nebenschäden „als eines Begriffs, der tote oder verwundete Zivilisten umschreibt, die sich besser eine sicherere Nachbarschaft gesucht hätten" - so das Time Magazine, - nicht unerheblich zum Zynismus der Berichterstattung bei. ${ }^{24}$

Von den Opfern, insbesondere unter der irakischen Zivilbevölkerung, war in den freigegebenen Berichten kaum die Rede. Diese Informationen wurden der Öffentlichkeit vorenthalten. Die Zensur hatte die Aufgabe, das Bild äußerster Präzision und damit der Ungefährlichkeit des Krieges zu vermitteln, mit Bravour erfüllt. Noch heute, fast ein Jahr nach Ende der Kampfhandlungen, sind die Folgen des Krieges nicht überschaubar. ${ }^{25}$ Das von der Propaganda gewünschte Kriegsbild hat sich in den Köpfen vieler Journalisten wie großer Teile der Bevölkerung festgesetzt. Die öffentliche Aufmerksamkeit gegenüber dem Geschehen im Kriegsgebiet am Persischen Golf ließ bereits während des Krieges nach. Der Krieg ist heute kein Thema mehr. Selbst der andauernde Versuch Saddams, das kurdische Volk im Norden und die schiitische Minderheit im Süden des Irak physisch zu vernichten, wird, wenn überhaupt, weitgehend ohne Bezug auf den Krieg behandelt.

Doch auch hier gilt, daß mit Sprache schon im Frieden manipuliert und zensiert wird. So sprach der frühere US-Präsident Ronald Reagan von „Friedensbewahrern“, als er stolz der Öffentlichkeit eine neue Atomrakete präsentierte. Kluge Bürokraten reden von „Entsorgungsparks“, wenn sie Atommülldeponien meinen. Arbeitgeber setzen Arbeitskräfte "frei“, wenn sie sie entlassen. Jeder Leser der morgendlichen Zeitung muß erst den alltäglichen Linguizid bewältigen, bevor er das tatsächliche Geschehen ans Tageslicht bekommt. 
toren nicht einfach erklären: „Die Militärzensur hat uns heute dieses oder jenes mitgeteilt. Mehr wissen wir nicht und auch nicht, ob es stimmt."

Marc Kravetz, Kolumnist der Pariser 'Libération', räumte selbstkritisch ein, daß er als schreibender Journalist, um konkurrenzfähig zu bleiben, viel zu oft die schnellen elektronischen Medien ungeprüft als Informationsquelle benutzt und damit zur 'Nachrichtenumweltverschmutzung' beigetragen habe. Seine Schlußfolgerung: „Wir müssen den Mut aufbringen, zu schreiben, daß wir nichts gesehen haben, wenn es so ist, daß wir halt nichts gesehen haben." 29

Wo waren die Aufrufe an Leser, Hörer und Zuschauer, bei der Bundesregierung, die die Beschneidung des Informationsrechtes durch die Alliierten wenn nicht unterstützte so doch zumindest duldete, zu protestieren? Wo waren die Aufforderungen an die Bundesregierung, für die Verteidigung der Presseund Informationsfreiheit offensiv einzutreten? Der DJV hat protestiert, die IGMedien sowie einzelne Redaktionsvertretungen, doch einmalige Demonstrationen bewirken nicht viel. Immer wieder gehören die Forderungen und Proteste in die eigenen Medien und auf die Tagesordnung politischer Auseinandersetzung. Wo sind die Proteste der Medien gegen die türkische Regierung, die mit Hilfe der politischen Polizei gewaltsam gegen Korrespondenten vor Ort und noch mehr gegen die kurdischen Journalisten vorgeht, die es wagen, entgegen den Verboten der Zensurbehörden über die Situation der türkischen Kurden zu berichten? Der von dem ARD-Korrespondenten in der Türkei Harald Weiss gezeigte scheinbare Sportsgeist zeugt dagegen eher von einer professionellen Deformation: „Denn je mehr die Regierenden (in Ankara - R. S.) zu verbergen suchen, desto neugieriger recherchieren die Journalisten. ... Solange das noch möglich ist, empfinden wir jede Behinderung unserer Arbeit mehr als Herausforderung denn als Versuch einer Zensur."30

\section{Zensur als permanentes Thema der Berichterstattung}

In aller Welt machen Journalisten Zensureingriffe mit weißer Fläche oder schwarzem Balken deutlich. Warum ist dies nicht auch bei der Zensur im Kriegsfall möglich? Was hindert die verantwortungsvollen Zeitungen, die Verlage, die Journalisten und ihre Verbände, sich zu einer solchen Boykottaktion zusammenzuschließen? Man mag diese Frage als naiv, als unprofessionell abtun; begründet beantwortet wurde sie bisher noch nicht. Zielstrebig wurde noch in fast jeder Diskussion einer kritischen Relexion darüber ausgewichen.

In die Praxis umsetzen ließe sich ein solcher Boykott dagegen recht einfach. Das Signal an Politik und Gesellschaft wäre unübersehbar. Dort, wo die zensierten Berichte zu plazieren wären, bliebe nur die Schlagzeile, die ankündigt, was hier eigentlich hätte stehen sollen. Statt des zensierten Textes druckt die Redaktion auf der freien Fläche eine Erklärung für den Leser und die Leserin ab, warum man auf dessen Abdruck lieber verzichtet. Das Gleiche gilt auch für Fotos. Anstatt strahlende Top-Gun-Helden oder saubere, unblutige Videobilder zu reproduzieren, erscheinen weiße Flächen. Auch hier würde eine Erklärung genügen: „Krieg ist blutig, schmutzig und nichts für Helden. Doch diese Bilder 
können und dürfen wir nicht zeigen, weil unsere Fotografen bis heute noch immer keine authentischen Fotos der Opfer aufnehmen und verbreiten durften!“

Doch mit einer einmaligen Aktion könnte das Problem der Zensur kaum dauerhaft präsent gemacht werden. Auf eine Berichterstattung völlig zu verzichten, bewirkt genau das Gegenteil dessen, was angestrebt ist. Der Krieg verschwindet dann aus dem öffentlichen Bewußtsein und die Auseinandersetzung um ihn käme sehr schnell zum Erliegen. Die Folge wäre genau das, was mit Zensur noch immer beabsichtigt war. Die Konsequenz kann aber nicht sein, den Boykott grundsätzlich als untauglich abzulehnen. Die Zeitungen könnten vielmehr rotierend den Boykott wiederholen. Die jeweils „normal“ erscheinenden Zeitungen hätten dann allerdings genauso wie die elektronischen Medien, die solidarische Pflicht, jeweils an herausragender Stelle über die Presseorgane, die sich im aktiven Boykott befinden, zu berichten.

Die Zensur nur zu beklagen reicht nicht. Dies würde schnell zum Alibi. Zensur jedweder Art muß angeprangert, ihre politischen Grundlagen müssen entzogen werden. Die Debatte darüber gilt es immer noch zu führen.

Anmerkungen:

1 Zur Geschichte der Militärzensur und den Entwicklungen seit dem Falkland-Krieg, vgl. Kurt Kister, Die Lust am Grauen, in: Süddeutsche Zeitung, 26./27.01.1991

2 U.S. Used Press as a Weapon, in: Wall Street Journal, 28.02.1991

3 Vgl. Frankfurter Rundschau, 04.03.1991, 1

4 Vgl. Operation Desert Shield Ground Rules, veröffentlicht durch das Pentagon am 14.1.1991, in: epd/Kirche und Rundfunk, Nr. 6, 26.01.1991, 26-27; ebenso die britischen „Media Ground Rules", die die Pool-Regelung allerdings nicht enthalten. Die Regeln liegen dem Verfasser in Fotokopie vor.

5 Vgl. Frankfurter Rundschau, 02.02.1991, 2

6 Vgl. medium, Nr. 2/91, 27.

7 International Herald Tribune, 14.02.1991, 5

8 Los Angeles Times, 14.01.1991, 4

9 Süddeutsche Zeitung, 22.02.1991, 1

10 Frankfurter Rundschau, Main-Kinzig-Ausgabe, 27.12.1991, 29

11 So befürworteten $57 \%$ der US-amerikanischen Bevölkerung nach Beginn der Kampfhandlungen eine weitere Verschärfung der Pressezensur. Vgl. epd/Kirche und Rundfunk, Nr. 9, 06.02.1991, 19

12 Time Magazine, 25.02.1991, 72

13 Vgl. zum verfassungs- und menschenrechtlichen Kontext, Hans-Joachim Reeb, Der Kampf um Informationen in Krisen und Kriegen, Koblenz 1991, 2-3

14 WDR-Sendemanuskript-Hörfunk, "Samstagabend im WDR III: Krieg am Golf - Krise im Journalismus" 01.06.1991, 21.

15 Peter Ludes/Georg Schütte, Militärische Optik. Die Invasion Kuwaits und der Krieg gegen den Irak, in: Tagesschau und heute, in: medium, Nr. 2/1991, 26

16 Vgl. Nicholas Hiley, Who won the Gulf reporting battle?, in: Times Literature Supplement, 22.03.1991; CNN paßte seine Anzeigenpreise umgehend der gestiegenen Zuschauerschaft an und verlangte für den gleichen 30-Sekunden-Spot mit 20.000 US-\$ rund $500 \%$ mehr als noch vor dem Krieg - damals etwa 3.500 US-\$. Vgl. Washington Journalist Review, März 1991, 28 
17 Vgl. Gisela Marx, Eine Zensur findet nicht statt, Reinbek (rororo Essay), 1989

18 Publizistik \& Kunst, Nr. 2, Februar 1991, 4; zu den USA vgl. „A Question Framed by The Boss", in: International Herald Tribune, 15.01.1991

19 Frankfurter Rundschau, 13.02.1991

$20 \mathrm{Vgl}$. epd/Kirche und Rundfunk, Nr. 9, 06.02.1991, 9 und 12

21 Vgl. FAIR/Extra!, Vol. 3, Nr. 8, Nov./Dez. 1990, 4 sowie Normon Solomon, „878 Voices, One for Peace: The Networks Call This Fair", in: International Herald Tribune, 25./26.05.1991, 5

22 Frankfurter Rundschau, Main-Kinzig-Ausgabe, 31.01.1991

23 Im November 1991 zeichnete die US-Nationalkonferenz der Englischlehrer das USVerteidigungsministerium für ihre „euphemistischen Erklärungen im Golf-Krieg“ mit dem an Orwells Sprachkritik erinnernden „Doublespeak“-Preis aus. Vgl. Frankfurter Rundschau, 25.11.1991, 1

24 Zit. bei Normon Solomon, siehe Fußnote 21; vgl. auch „Tote als 'Nebenschaden'“, in: Frankfurter Rundschau, 12.02.1991, 2

25 Vgl. hierzu u. a. Naturwissenschaftler-Initiative "Verantwortung für den Frieden", Eine vorläufige Bilanz des Golfkrieges, Hamburg 1991; Michael Müller, „Die Golfregion nach dem Krieg: Ein Ökologisches Desaster", in: Georg Stein (Hg.), Nachgedanken zum Golfkrieg, Heidelberg 1991, 260-273; Ulrich Gottstein, „Der Irak nach dem Krieg: Ein zerschlagenes Land", in: ebd., 274-278

26 Vgl. Der unsichtbare Krieg, in: Neue Zürcher Zeitung, Fernausgabe, 10./11.02.1991; außerdem ausführlich zur Ölpest und den brennenden Ölquellen: Der Krieg in den Medien, Zensur fand (nicht) statt, in: Naturwissenschaftler-Initiative 'Verantwortung für den Frieden', Eine vorläufige Bilanz des Golfkrieges, Hamburg 1991, 16-17. Zur Feindbildproblematik im Golf-Krieg vgl. Dossier „Feindbilder“, in: Informationsdienst der Informationsștelle Wissenschaft und Frieden, Nr. 3/91; Reinhard Schulze, „Alte und neue Feindbilder. Das Bild der arabischen Welt und des Islam im Westen“, in: Georg. Stein (Hg.), Nachgedanken zum Golfkrieg, Heidelberg 1991, 244-259 sowie Jürgen Link, „Der irre Saddam setzt seinen Krummdolch an meine Gurgel", Frankfurter Rundschau-Dokumentation, 28.01.1991, 9

27 Vgl. Insight, 18.02.1991, 17; zu den Umfrageergebnissen vgl. epd/Kirche und Rundfunk, Nr. 9, 6.2.1

$28 \mathrm{Vgl}$. den kritischen Bericht von Marcel Pott, ARD-Korrespondent in Amman, in: WDRSendemanuskript-Hörfunk, siehe Fußnote 14, 24-27

29 Vgl. Frankfurter Rundschau, 21.02.1991, 18

30 SWF-Journal, Nr. 3, März 1991, 15 


\section{SUMMARY}

Besides the question whether or not a military intervention in order to liberate Kuwait from the Iraqi occupation would be legitimate at all, one of the most controversial measures of the entire war was the military censorship, extensively practiced by all involved nations, and its purpose. The author describes the rules of censorship and its practical consequences for the freedom of press correspondence. He generally denies the legitimacy of state-controlled censorship in a democracy, for whatever reason. In his opinion, democracy and censorship exclude each other. But also the role of the media, its willingness to submit to the military censorship more or less uncritically, is being criticised severely. According to the hypothesis of the author, the renunciation of resistance against the censorship, whether it be out of opportunism, convenience, interest in economic exploitation or compulsion to do so, means nothing else, but the abandoning of the right of free journalism and free access to information. Concluding, the author proposes some actions to be undertaken by the media in case of state-controlled measures of censorship being repeated in the future, and leaves them open for discussion.

\section{RÉSUMÉ}

Indépendanment de la question si une intervention militaire pouvait être légitime afin de libérer le Kuwait de l'occupation irakienne, c'est la censure militaire, (ainsi que sa fonction), Pratiquée de façon extensive par tous les états, participants, qui fut la mesure la plus contestée de toute la guerre. L'auteur décrit les règles de censure et leurs conséquences pour la pratique du reportage libre. Quelle qu'en soit l'utilité, il conteste le principe de la légitmité d'une censure d'Etat en démocratie. D'après lui, démocratie et censure ne sont pas compatibles. Et le rôle de médias, qui acceptèrent presque sans broncher de se soumettre à la censure militaire, est aussi vivement critiquable. Il y va non seulement d'avoir suivi les mesures censoriales, mais également d'avoir pratiqué une autocensure anticipée et multiple dans presque tous les domaines de l'information. Renoncer à combattre la censure, soit par opportunisme, par confort, par intérêts (ou contraintes) économiques n'est, telle est la thèse de l'auteur,rien d'autre que l'abandon pour et simple du droit au journalisme libre et à l'accès aux informations. Pour terminer, l'auteur suggère un débat sur quelques propositions, d'actions de médias, au cas où l'Etat recidiverait à établir des formes de censure.

\section{RESUMEN}

Junto a la pregunta de si la intervención militar para la liberación militar de Kuwait de la ocupación iraquí podía ser legítima, una de las más controvertidas medidas de toda la guerra fue la censura militar y su función, practicada en extenso por todos los países involucrados. El autor describe las reglas de censura y sus consecuencias para la práctica de un periodismo libre. El impugna básicamente la legitimidad de una censura por parte del estado en una democracia, sea cual sea el motivo. Según su opinión democracia y censura se excluyen mutuamente. También el rol de los medios de comunícación, su disposición a someterse tan sin crítica a la censura militar, es sometido a fuertes críticas. La renuncia a realizar una resistencia contra la censura, sea por oportunismo, por comodidad o por intereses económicos ou obligaciones económicas significa, según la tesis del autor, no otra cosa que la renuncia del derecho a un periodismo libre y a acceso a información. Para concluir el autor pone a discusión algunas propuestas para acciones de los medios de comunicación caso de repetición, en el futuro, de medidas de censura por parte del estado. 